Laser Chem., 1999, Vol. 19, pp. 371-374

Reprints available directly from the publisher Photocopying permitted by license only
(C) 1999 OPA (Overseas Publishers Association) N.V. Published by license under the Harwood Academic Publishers imprint, part of The Gordon and Breach Publishing Group.

\title{
FEMTOSECOND TRANSIENT ABSORPTION STUDIES OF SIMPLE POLYENES IN SOLUTION
}

\author{
KAORU OHTA $^{\mathrm{a}, \mathrm{b}, *}$, YUKITO NAITOH ${ }^{\mathrm{b}}$, \\ KEISUKE TOMINAGA $^{\mathrm{b}}$, NOBORU HIROTA ${ }^{\mathrm{a}}$ \\ and KEITARO YOSHIHARA ${ }^{\mathrm{b}, *}$ \\ a Department of Chemistry, Kyoto University, Kyoto 606, Japan; \\ ${ }^{\mathrm{b}}$ Institute for Molecular Science, Myodaiji, Okazaki 444, Japan
}

(Received 8 April 1997)

We have conducted femtosecond transient absorption experiments on the excited-state dynamics of trans- and cis-hexatriene (HT) in solution. The transient absorption in the visible region decays with the time constant of about $500 \mathrm{fs}$, indicating that the internal conversion (IC) to the ground state occurs quite efficiently. The bleach recovery signal contains several time constants. The vibrational relaxation in the ground state occurs in $10-20 \mathrm{ps}$ and the slow relaxation process, which may be due to the conformational change around $\mathrm{C}-\mathrm{C}$ single bond, proceeds in a subnanosecond time scale.

Keywords: Transient absorption; hexatriene; excited-state dynamics; vibrational relaxation

\section{INTRODUCTION}

The photochemistry of small linear polyenes has stimulated a great deal of interest over the past two decades as a simple model for more complex systems such as retinals and carotenoids. However, the excited-state properties of HT have not been well understood because of a complete lack of fluorescence and broad absorption spectra even under a jet-cooled condition. In order to directly reveal the excited-

\footnotetext{
${ }^{*}$ Corresponding authors.
} 
state dynamics in solution, we have performed the ultrafast transient absorption measurement of trans- and cis- HT in cyclohexane and acetonitrile solutions $[1,2]$.

\section{EXPERIMENTAL}

Both isomers of HT were pumped by the third harmonic of a regeneratively amplified $\mathrm{Ti}$ :Sapphire laser which produced a $120 \mathrm{fs}$ pulse at around $800 \mathrm{~nm}$. The transient absorption and bleach recovery were probed by using a white light continuum and third harmonic, respectively.

\section{RESULTS AND DISCUSSION}

Figure 1 shows the transient absorption signals in cyclohexane probed at three different wavelengths. They consist of an instrument-limited rise $(<150 \mathrm{fs})$ and subpicosecond decay at each probe wavelength. For trans-HT, the signal observed at $400 \mathrm{~nm}$ decays with a time constant of about $400 \mathrm{fs}$. At longer wavelengths the decay of the signal
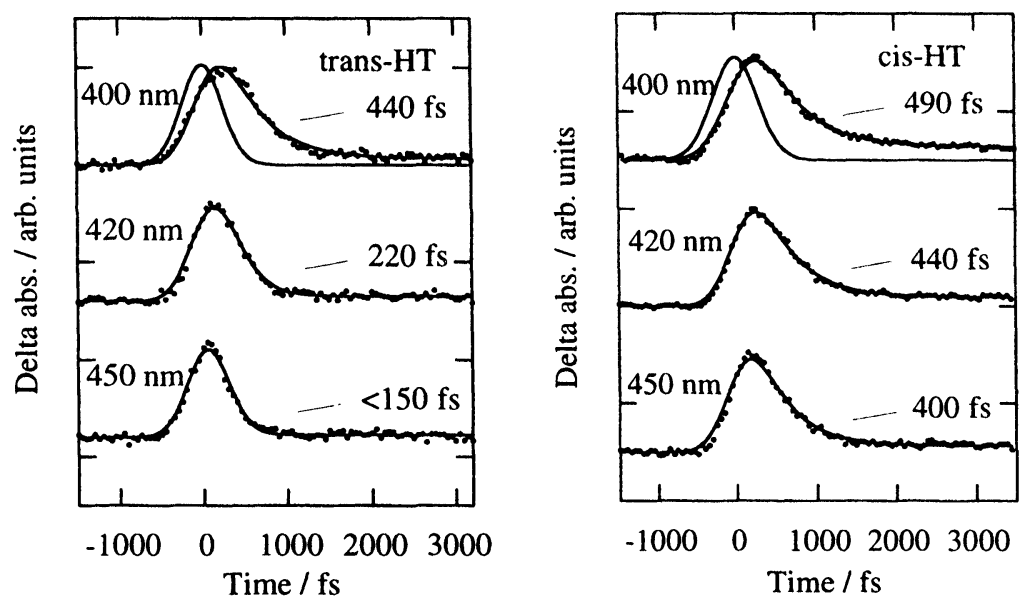

FIGURE 1 Transient absorption signals probed at three different wavelengths in cyclohexane. The instrument response function is shown in the top decay curve. 
gradually becomes faster and approaches the instrument response function at probe wavelengths longer than $450 \mathrm{~nm}$. On the other hand, the temporal profiles of cis-HT do not depend on the probe wavelength significantly. The transient absorption signals in acetonitrile are similar to those in cyclohexane.

It is considered that the photoexcitation to the $S_{2}$ state is followed by the IC to the $S_{1}$ state which occurs within $100 \mathrm{fs}[3,4]$. The probe wavelength dependent decay of the transient absorption is ascribed to the vibrational energy redistribution (VER) in the $S_{1}$ state and the IC to the $\mathrm{S}_{0}$ state. The difference observed in trans- and cis- HT may depend on which contribution is larger in the two competitive processes.

Figure 2 shows the one-color transient bleach recovery signals at $268 \mathrm{~nm}$. The transient exhibits an instrument-limited appearance of a bleach followed by the recovery which occurs in several time scales.
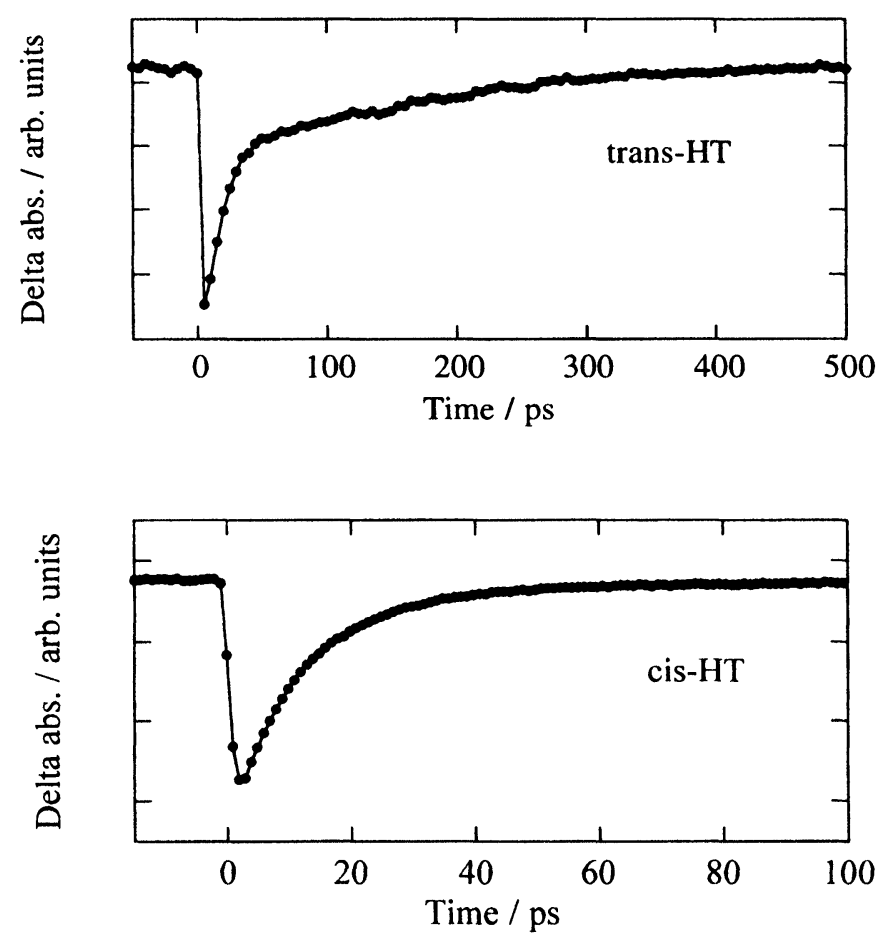

FIGURE 2 Transient bleach recovery signals at $268 \mathrm{~nm}$ in cyclohexane. 
The temporal profile of trans-HT in the first $20 \mathrm{ps}$ is similar to that of cis-HT. However, in a longer time scale, these signals are quite different to each other. While, in trans-HT, it takes $150-300$ ps to fully recover the bleach, cis-HT needs almost no more than $100 \mathrm{ps}$.

The transient bleach signal within the first 20 ps shows that it takes about $1 \mathrm{ps}$ to observe the population of the vibrationally excited $\mathrm{S}_{0}$ state at $268 \mathrm{~nm}$ and the subsequent vibrational relaxation in the $S_{0}$ state occurs in $10-20 \mathrm{ps}$ [5].

The low quantum yield of trans-cis photoisomerization of the $\mathrm{C}=\mathrm{C}$ double bond in solution means that recovery process to the original form dominates in the signal. One plausible origin of the 100ps component is a conformational change around the $\mathrm{C}-\mathrm{C}$ single bond. After photoexcitation, s-cis isomer is produced in the excited state or in the subsequent vibrationally excited ground state. The thermal redistribution among different $s$-cis isomers occurs in a subnanosecond time scale. The different behavior between trans- and cis- HT may reflect different product distribution and dynamics among $s$-cis isomers.

\section{References}

[1] Ohta, K. et al. (1996). Chem. Phys. Lett., 256, 629.

[2] Ohta, K. et al. (1998). J. Phys. Chem. A, 102, 35.

[3] Hayden, C. C. and Chandler, D. W. (1995). J. Phys. Chem., 99, 7897.

[4] Ci, X. et al. (1990). J. Chem. Phys., 92, 4708.

[5] Reid, P. J. et al. (1993). J. Am. Chem. Soc., 115, 4754. 